\title{
Expression and potential role of chemokine receptor CXCR4 in human bladder carcinoma cell lines with different metastatic ability
}

\author{
HAIFENG WANG, DELIN YANG, KAI WANG and JIANSONG WANG \\ Department of Urology, Second Affiliated Hospital of Kunming Medical College, Kunming 650101, P.R. China
}

Received October 18, 2010; Accepted February 14, 2011

DOI: $10.3892 / \mathrm{mmr} .2011 .440$

\begin{abstract}
The aim of the present study was to investigate the expression of the chemokine receptor CXCR4 in human bladder carcinoma cells and its possible role in their metastatic growth in vitro. Immunofluorescent imaging and flow cytometry were performed to examine the expression of CXCR4 in the EJ and EJ-M3 cell lines. A highly specific CXCR4 antagonist, AMD3100, was used to inhibit metastatic growth, as assessed by an in vitro invasion assay. The expression of CXCR4 protein was mainly observed in the cytoplasm of the two bladder carcinoma cell sublines. CXCR4 expression was increased in the EJ-M3 cell line compared to the EJ cells. Differences in the CXCR4 protein expression rates between the EJ-M3 cell line $(55.7 \pm 10.35 \%)$ and EJ cell line $(25.4 \pm 4.69 \%)$ were significant $(\mathrm{P}<0.05)$. The CXCR4 antagonist AMD3100 partially inhibited the metastasis of the EJ-M3 cells, which had higher CXCR4 expression $(56 \pm 8.96)$ than the control EJ cells $(118 \pm 6.56)(\mathrm{P}<0.05)$. In conclusion, the high in vitro metastatic potential of human bladder carcinoma cell sublines is closely associated with increased CXCR4 expression, and is inhibited by a specific CXCR4 antagonist.
\end{abstract}

\section{Introduction}

Bladder carcinoma is a common genitourinary cancer, and the malignant properties of its recurrence and metastasis make it resistant to clinical treatment. However, the mechanisms of the invasive growth of bladder carcinoma remain unclear.

CXCR4 is a G protein-coupled receptor that comprises seven transmembrane domains. Recent research has revealed that this protein is also a co-receptor for foreign HIV. Stem cell-derived factor $\alpha(\mathrm{SDF}-1 \alpha)$, the natural ligand for CXCR4,

Correspondence to: Dr Delin Yang, Department of Urology, Second Affiliated Hospital of Kunming Medical College, Kunming 650101, P.R. China

E-mail: wanghaifeng0871@gmail.com

Key words: bladder carcinoma, chemokine receptor, metastatic potential is a member of the CXC chemokine family, which has chemotactic activity for hematopoietic progenitor cells (1-4). Chemokine signaling results in the transcription of the target genes involved in cell invasion, motility, interaction with the extracellular matrix (ECM) and survival (5). The expression of the chemokine receptor CXCR4 plays a key role in migration and metastasis, and is associated with tumor progression and poor prognosis in a number of malignancies (6-9). We detected and analyzed the expression of CXCR4 in the bladder cancer cell line EJ and the highly invasive bladder carcinoma cell line EJ-M3 in order to investigate the role of CXCR4 in the invasion and metastasis of bladder cancer.

\section{Materials and methods}

Cell lines and antibodies. The highly invasive human bladder carcinoma EJ-M3 cell line was established in a previous experiment (10). The EJ-M3 cell line and the parent bladder carcinoma EJ cell line were cultured in DMEM supplemented with $10 \%$ fetal bovine serum and incubated in $5 \% \mathrm{CO}_{2} / 95 \%$ air at $37^{\circ} \mathrm{C}$. A routine immunostaining procedure was then performed with CXCR4 multi anti-rabbit IgG (G-19) (Santa Cruz, USA) as the primary antibody, Alexa Fluor 594 donkey anti-rabbit IgG $(\mathrm{H}+\mathrm{L})$ and mouse anti-human CXCR4 as the secondary antibodies, goat anti-mouse monoclonal antibody as the control, and AMD3100 as the CXCR4 antagonist (all from Invitrogen, USA). Antibody solutions were diluted in phosphate-buffered saline (PBS) at a concentration of $3 \mathrm{ml} /$ well.

Immunofluorescence analysis. An indirect immunofluorescence technique was used to detect the expression of CXCR4 protein in the EJ and EJ-M3 cell lines. In brief, bladder cancer cells in the logarithmic phase of growth were suspended in medium at a concentration of $1 \times 10^{4} / \mathrm{ml}$ and seeded overnight on sterile coverslips placed into a 6 -well plate. The medium was removed and the cells were washed twice with PBS, then fixed in methanol at $4^{\circ} \mathrm{C}$ for $10 \mathrm{~min}$. The coverslips were washed with $0.25 \%$ Triton $\mathrm{X}-100$ and $1 \%$ BSA. The first and second antibody staining were performed in buffered glycerol. PBS was used instead of the first antibody as a negative control. Staining was observed using a fluorescence microscope (Nikon, Japan). Images were captured using a multispectral imaging system (CRI). 

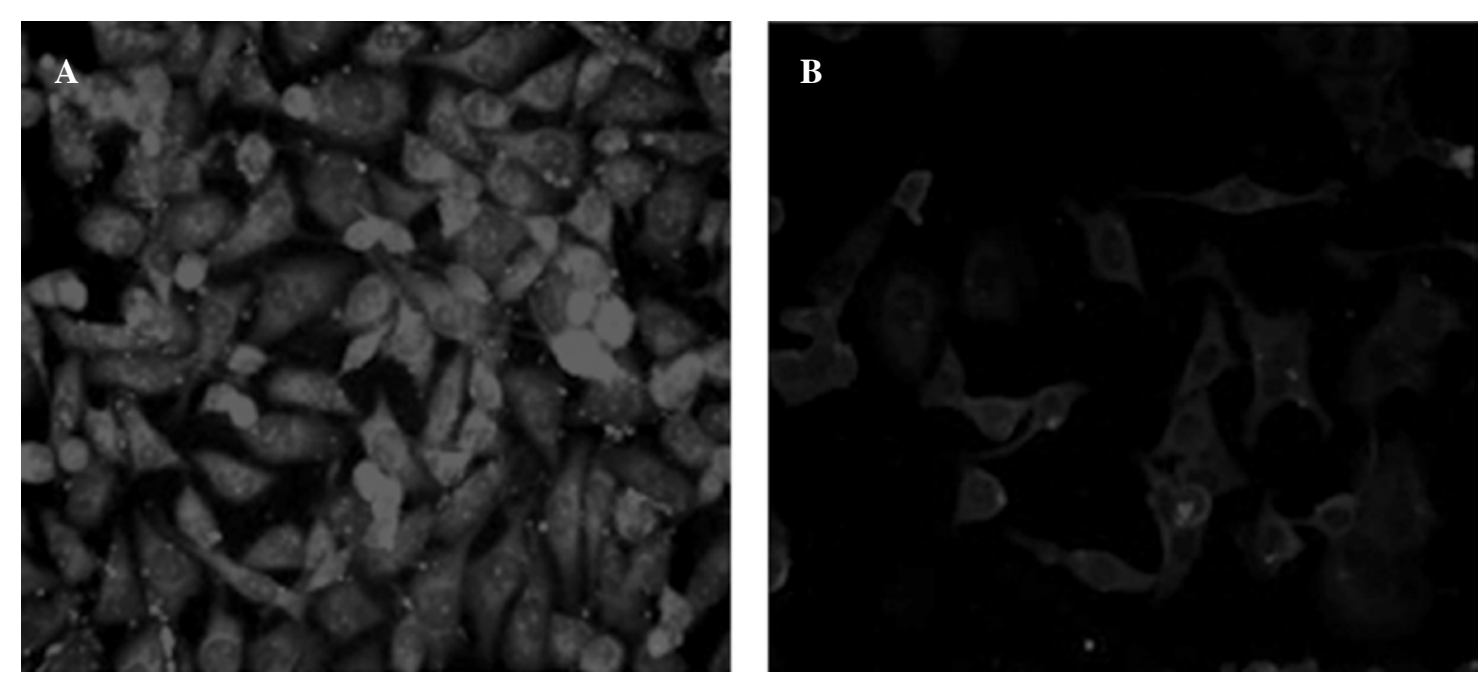

Figure 1. Immunofluorescence microscopy analysis of CXCR4 expression in the EJ-M3 (A) and EJ (B) cell lines. The EJ-M3 cell line exhibited stronger staining of CXCR4 protein than the EJ cell line.

Flow cytometric analysis. To confirm the expression of CXCR4 protein in the EJ and EJ-M3 cell lines, flow cytometry was performed. In brief, bladder cancer cells in the logarithmic phase of growth were trypsinized on ice for $10 \mathrm{~min}$ and fixed in $4 \%(\mathrm{w} / \mathrm{v})$ paraformaldehyde. The cells were washed and incubated with anti-human CXCR4 mAb 12G5 (1:100 dilution) or mouse IgG isotype control for $30 \mathrm{~min}$ at room temperature. After being washed twice with PBS, the cells were incubated with PE-labeled goat anti-mouse IgG for $30 \mathrm{~min}$ at room temperature and analyzed on an Epics flow cytometer (Becton Dickinson, USA).

Migration assay using AMD3100. To determine the effect of CXCR4 on the metastatic growth of the EJ-M3 cells, cell migrations assays were conducted in cells treated with or without the AMD3100 antagonist. The assays were performed using 6.5-mm-diameter Transwell ${ }^{\mathrm{TM}}$ inserts with uncoated polycarbonate filters (5- $\mu \mathrm{m}$ pore size) (Costar, USA). Briefly, before adding cells to the upper compartment, the transwells were washed 3 times with RPMI 1640 medium plus $0.25 \%$ BSA, provided with the assay. EJ-M3 cells $\left(1 \times 10^{5}\right)$ were resuspended in $0.1 \mathrm{ml}$ assay medium and seeded in the upper compartment of the transwells. Medium at a volume of $0.6 \mathrm{ml}$ with or without $100 \mathrm{ng} / \mathrm{ml} \mathrm{SDF}-1 \alpha$ was added to the lower compartment. A 0.1 -ml cell-containing sample was maintained in the assay medium as an input control for the quantitation of the number of migrated cells. Cells were then left untreated or treated with the addition of $100 \mathrm{ng} / \mathrm{ml} \mathrm{AMD} 3100$ at $37^{\circ} \mathrm{C}$ in $5 \% \mathrm{CO}_{2}$ for $12 \mathrm{~h}$. Subsequently, the inserts were removed, non-invasive cells were wiped off the upper surface of the filter with a moistened cotton swab, and cells that had invaded onto the lower surface of the membrane were fixed in methanol for $30 \mathrm{~min}$, then stained with H\&E. Migrated cells were counted using an inverted microscope (Olympus, Japan). The membranes were divided into four quadrants by horizontal and vertical lines.

Statistical analysis. The experimental data were analyzed with SPSS 10.0 statistical software, with the level of signifi- cance set at $\mathrm{P}<0.05$. All experiments were repeated two to three times, and similar results were obtained.

\section{Results}

Immunofluorescence. CXCR4 protein was stained light red in the EJ cells and red or dark red in the EJ-M3 cells, indicating that it was expressed more strongly in the EJ-M3 cells compared to the EJ cells (Fig. 1).

Flow cytometry. In accordance with the immunofluorescence results, flow cytometric data indicated that $55.7 \pm 10.35 \%$ of the EJ-M3 cells were positive for CXCR4 expression, while only $25.4 \pm 4.69 \%$ of EJ cells were positive for CXCR4. The expression of CXCR4 protein was significantly higher in the EJ-M3 cells compared to the EJ cells, as determined by the t-test (Fig. 2).

Migration assay AMD3100. In the EJ-M3 cells treated with AMD3100, the number of invasive cells was $56 \pm 8.96$. This was significantly lower than the number of cells in the nonintervention group (118 \pm 6.56$)$. (Fig. 3).

\section{Discussion}

The mechanisms of cancer metastasis are complex, and new discoveries have led to the expansion of the 'seed and soil' hypothesis. After metastatic cells pass through vascular channels and are implanted in the bladder, a favorable 'soil' may prompt further growth and proliferation. The establishment of bladder metastasis results from a close interaction between metastatic tumor cells and the unique environment of the bladder.

The formation of aggressive bladder metastases is closely associated with a distinctive gene expression profile (11). The chemokine receptor CXCR4 has been considered one of the mechanisms related to bladder tumor cell metastasis. Tumors that express higher levels of CXCR4 have been found to be 

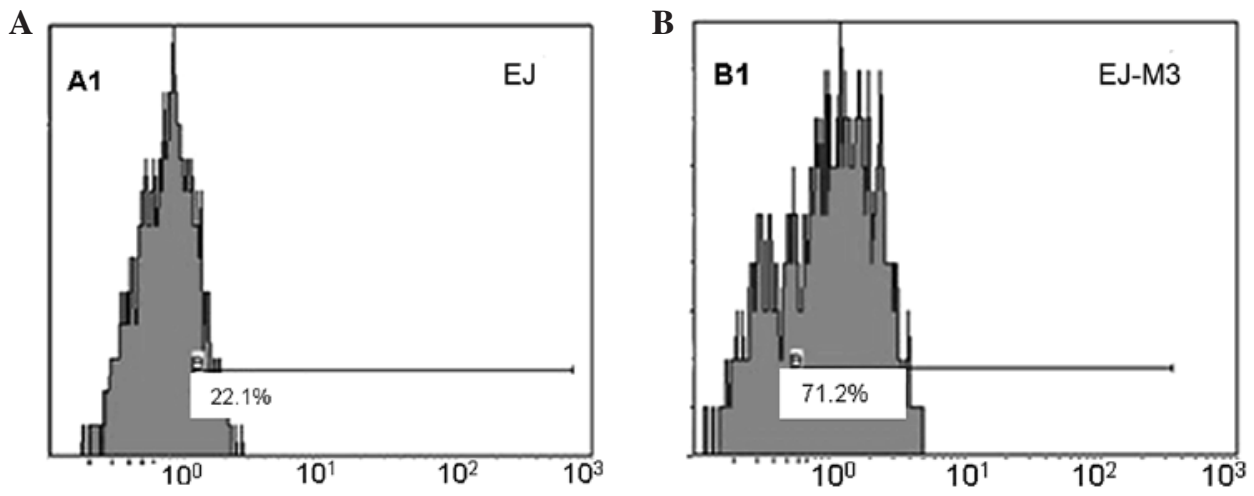

FL1 LOG
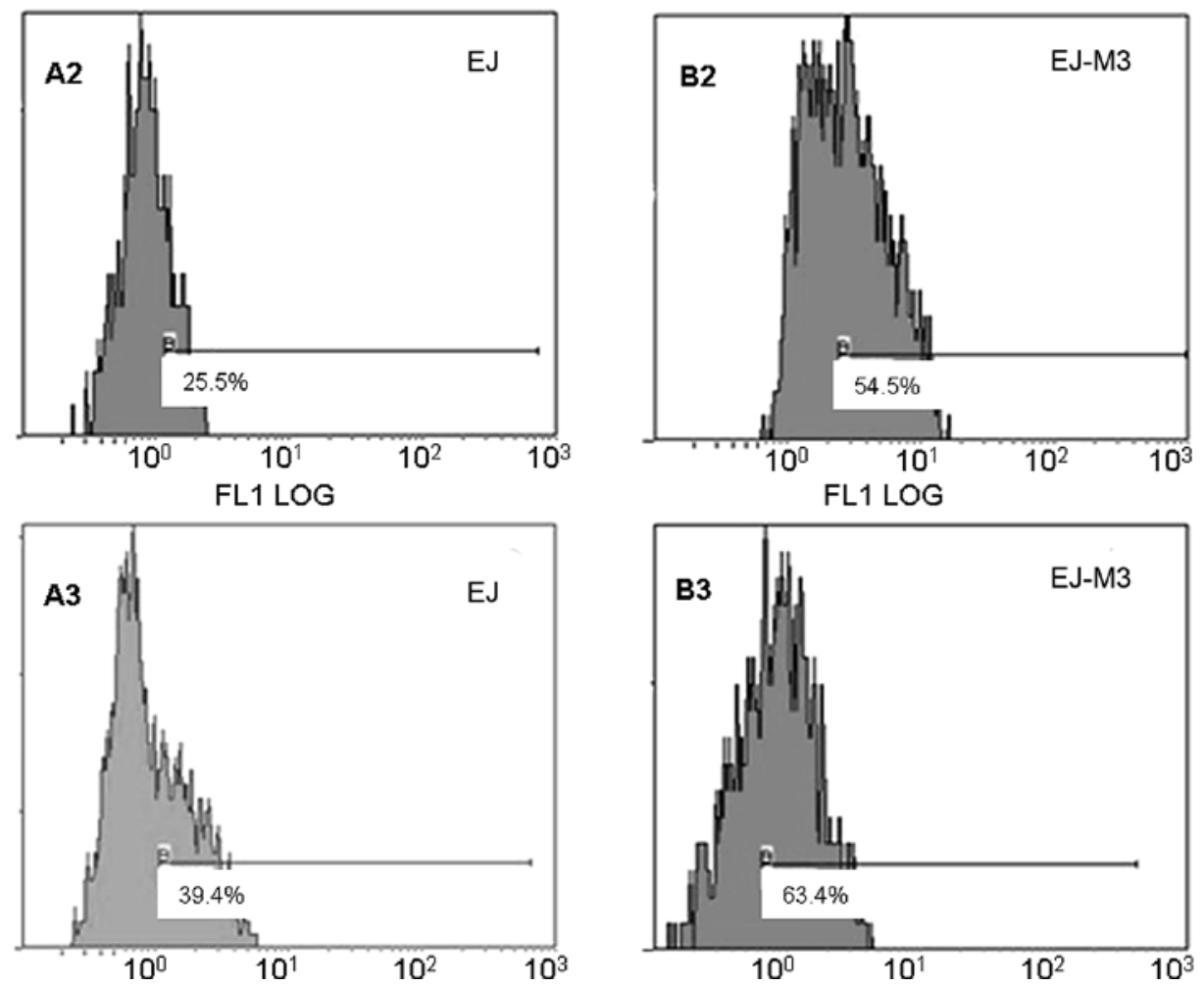

Figure 2. Flow cytometric analysis of CXCR4 expression in the EJ (A) and EJ-M3 (B) cell lines. The percentage of cells positive for CXCR4 expression was significantly higher in the EJ-M3 cell line than in the EJ cell line $(\mathrm{P}<0.05)$.
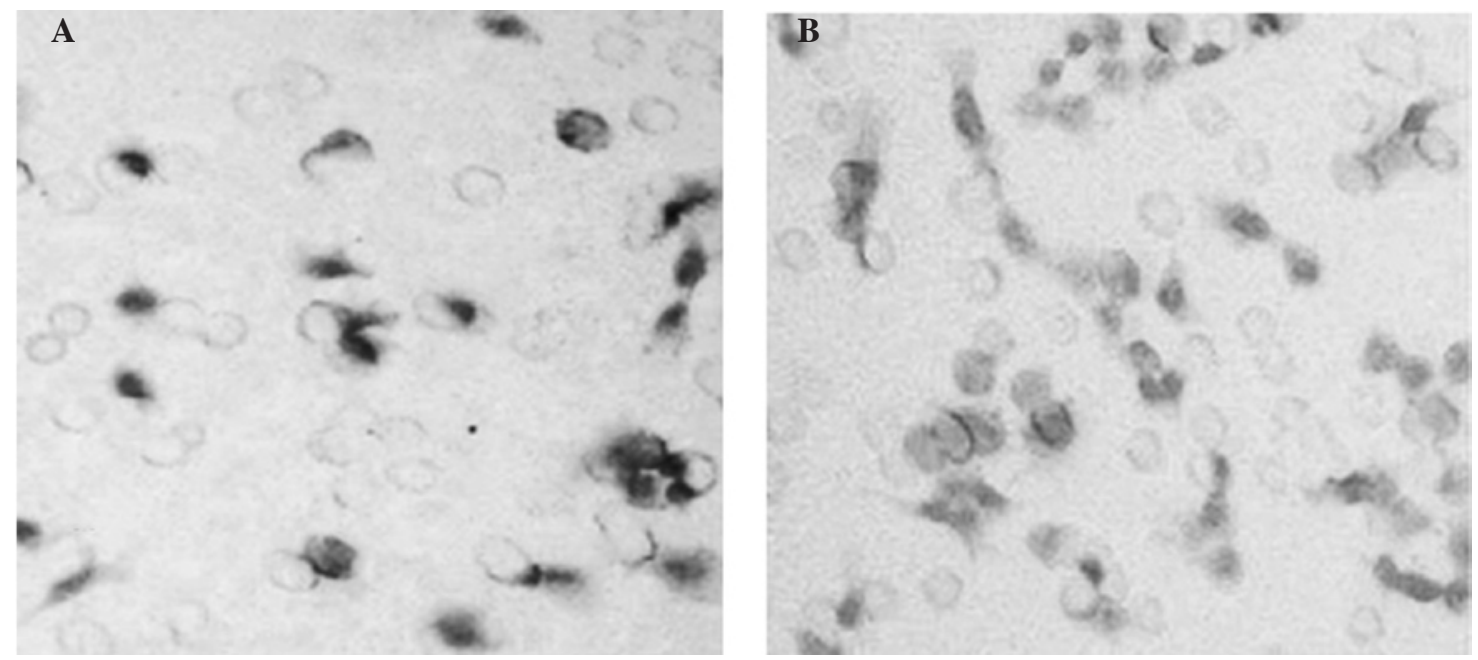

Figure 3. Migration assay of EJM3 cells treated with (A) or without (B) AMD3100 antagonist. The number of invasive cells was significantly lower in the group treated with AMD3100 compared to the untreated group $(\mathrm{P}<0.05)$. 
more prone to cancer metastasis than those with lower expression $(12,13)$. It is well known that peripheral lymphocytes preferentially localize to peripheral lymphoid tissues, such as the lymph nodes; this is called the homing phenomenon (14). Evidence suggests that CXCR4 overexpression in metastatic cancer cells plays an important role in the homing of the cells (15). CXCR4 is a natural receptor for SDF-1, which has potent chemotactic activity for lymphocyte participation in directing the metastasis of these cells to the bladder, which expresses high levels of SDF-1 (16). High levels of SDF-1 are present in organs that are commonly invaded by metastasizing cells, and these high SDF-1 levels attract CXCR4-positive tumor cells. The interaction between SDF-1 and CXCR4 activates the specific signaling pathway controlling homing and metastasis. CXCR4 overexpression was found to enhance bladder tumor growth (17). In addition, CXCR4 may enhance invasive signals and metastatic growth in the bladder microenvironment. CXCR4 mRNA expression in normal human urothelium, bladder cancer and also bladder cancer cell lines (J82 and T24) was previously reported (18). The addition of SDF-1 (which presumably activates CXCR4 receptors) was found to increase Matrigel invasion and cell growth, but was not effective in increasing intracellular calcium in these particular urothelial cancer cells (18).

In the present study, both the EJ and EJ-M3 cell lines expressed CXCR4. The percentage of CXCR4-positive cells in the EJ-M3 cell line was significantly higher than that in the EJ cell line. This difference was statistically significant. Differences were noted in regards to in vitro invasion between the bladder cancer cell lines exhibiting differential expression of CXCR4, and the expression of CXCR4 was positively correlated with tumor invasion. This suggests that the SDF-1/CXCR4 biological axis plays an important role in tumor invasion and metastasis of bladder cancer. The high expression of CXCR4 was associated with a high invasive and metastatic potential of bladder cancer in vitro. This may provide clues to the prognosis and treatment of patients with bladder cancer.

In this study, we observed that AMD3100, a CXCR4 antagonist, inhibited bladder cancer cell invasion in vitro. This finding confirmed that blocking the expression of CXCR4 specificity reduces bladder cancer cell invasion in vitro. Additionally, it confirmed the close relationship between CXCR4 expression and the invasion of bladder tumor cell lines in vitro. This study provides new insights for the elucidation of the molecular mechanisms involved in the impact of the SDF-1/CXCR4 biological axis on the invasiveness and metastasis of bladder cancer, and suggests a new target for the control of bladder cancer metastasis.

\section{References}

1. Nagasawa T, Nakajima T Tachibana K, et al: Molecular cloning and characterization of a murine pre-B-cell growth-stimulating factor/stromal cell-derived factor 1 receptor, a murine homolog of the human immunodeficiency virus 1 entry coreceptor fusin. Proc Natl Acad Sci USA 93: 14726-14729, 1996.

2. Nagasawa T, Kikutani H and Kishimoto T: Molecular cloning and structure of a pre-B-cell growth-stimulating factor. Proc Natl Acad Sci USA 91: 2305-2309, 1994.

3. Aiuti A, Webb IJ, Bleul C, Springer T and Gutierrez-Ramos JC: The chemokine SDF-1 is a chemoattractant for human CD34+ hematopoietic progenitor cells and provides a new mechanism to explain the mobilization of $\mathrm{CD} 34^{+}$progenitors to peripheral blood. J Exp Med 185: 111-120, 1997.

4. Jo DY, Rafii S, Hamada T and Moore MA: Chemotaxis of primitive hematopoietic cells in response to stromal cell-derived factor-1. J Clin Investig 105: 101-111, 2000.

5. Locati M, Deuschle U, Massardi ML, et al: Analysis of the gene expression profile activated by the $\mathrm{CC}$ chemokine ligand 5/ RANTES and by lipopolysaccharide in human monocytes. J Immunol 168: 3557-3562, 2002.

6. Schimanski CC, Schwald S, Simiantonaki N, et al: Effect of chemokine receptors CXCR4 and CCR7 on the metastatic behaviour of human colorectal cancer. Clin Cancer Res 11: 1743-1750, 2005.

7. Kato M, Kitayama J, Kazama $\mathrm{S}$ and Nagawa H: Expression pattern of CXC chemokine receptor-4 is correlated with lymph node metastases in human invasive ductal carcinoma. Breast Cancer Res 5: R144-R150, 2003.

8. Uchida D, Begum NM, Almofti A, et al: Possible role of stromal-cell-derived factor-1/CXCR4 signaling on lymph node metastasis of oral squamous cell carcinoma. Exp Cell Res 290: 289-302, 2003.

9. Scotton CJ, Wilson JL, Milliken D, Stamp G and Balkwill FR: Epithelial cancer cell migration: a role for chemokine receptors? Cancer Res 61: 4961-4965, 2001.

10. Zhang C, Yang D, Wang J, Xu H: Isolation and identification of human bladder cancer cell line with high invasiveness. J Xinxiang Med College 25: 130-133, 2008.

11. Kang Y, Siegel PM, Shu W, et al: A multigenic program mediating breast cancer metastasis to bone. Cancer Cell 3: 537-549, 2003.

12. Su L, Zhang J, Xu H, Wang Y, Chu Y, Liu R and Xiong S: Differential expression of CXCR4 is associated with the metastatic potential of human non-small cell lung cancer cells. Clin Cancer Res 11: 8273-8280, 2005.

13. Hu J, Deng X, Bian X, et al: The expression of functional chemokine receptor CXCR4 is associated with the metastatic potential of human nasopharyngeal carcinoma. Clin Cancer Res 11: 4658-4665, 2005.

14. Balkwill F: Cancer and the chemokine network. Nat Rev Cancer 4: 540-550, 2004.

15. Muller A, Homey B, Soto H, et al: Involvement of chemokine receptors in breast cancer metastasis. Nature 410: 50-56, 2001.

16. Kucia M, Reca R, Miekus K, et al: Trafficking of normal stem cells and metastasis of cancer stem cells involve similar mechanisms: pivotal role of the SDF-1-CXCR4 axis. Stem Cells 23: 879-894, 2005.

17. Chinni SR, Yamamoto H, Dong Z, Sabbota A, Bonfil RD and Cher ML: CXCL12/CXCR4 transactivates HER2 in lipid rafts of prostate cancer cells and promotes growth of metastatic deposits in bone. Mol Cancer Res 6: 446-457, 2008.

18. Eisenhardt A, Frey U, Tack M, et al: Expression analysis and potential functional role of the CXCR4 chemokine receptor in bladder cancer. Eur Urol 47: 111-117, 2005. 